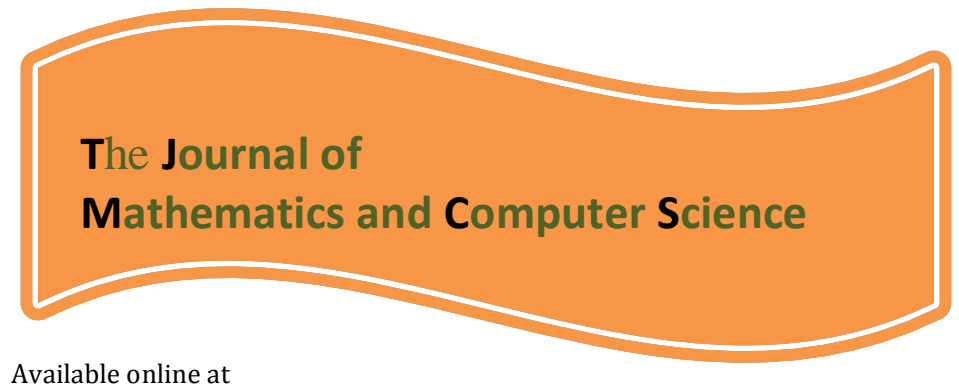

http://www.TIMCS.com

The Journal of Mathematics and Computer Science Vol .2 No.4 (2011) 672-681

\title{
Application of Adomian Decomposition Method for Solving Impulsive Differential Equations
}

\author{
H. Hossainzadeh ${ }^{1}$, G. A. Afrouzi ${ }^{1}$ and A. Yazdani ${ }^{1}{ }^{*}$ \\ 1 Department of Mathematics, Faculty of Mathematical Sciences, University of \\ Mazandaran, Babolsar, Iran \\ yazdani@umz.ac.ir \\ afrouzi@umz.ac.ir
}

Received: August 2010, Revised: November 2010

Online Publication: January 2011

\section{Abstract}

In this work, we apply the Adomian Decomposition Method(ADM) for solving first order impulsive differential equations

$$
\begin{aligned}
& \dot{x}(t)=\alpha x, t \neq k, t>0, \\
& \Delta x=\beta x, t=k, \\
& x\left(0^{+}\right)=x_{0},
\end{aligned}
$$

where $\alpha \neq 0, \beta, x_{0} \in \mathrm{R}, 1+\beta \neq 0, k \in \mathrm{N}$ are investigated. We compare this method with others numerical methods such as $\theta$-method, Runge-kutta method for solving impulsive differential equations.

Keywords: Impulsive differential equations; Adomian Decomposition Method; $\theta$-method ;Runge-Kutta method.

2000 AMS Subject Classification: Primary 34A37; Secondary 74H15.

\footnotetext{
* Corresponding author
} 


\section{Introduction and preliminaries}

Impulsive differential equations occur in many applications: population dynamics[1], physics , Chemistry [2], engineering[3], ecology, biological systems, biotechnology, industrial robotics, pharmacokinetics, optimal control, and so on. The quantitive investigation of impulsive differential equations began in 1960 with the work of Mil'man and Myshkis[4]. In recent years, there have been intensive studies on the qualitative behavior of solutions of impulsive differential equations; see for instance $[4,5,6,7,8,9]$ and the references cited therein. In 1989, Kulev and Bainov investigated the stability and global stability of systems with impulse by Lyapunov function [10,11]. In 2000, Randelovic gave the algorithm for solving impulsive differential equations [12]. However, in these works the authors did not investigate the stability of the numerical methods for impulsive differential equations. X. J. Ran et al. introduced some basic knowledge of the system with impulsive effect at fixed instant of time[13].

We shall apply the Adomian Decomposition Method (ADM) for solving the following system

$$
\begin{aligned}
& \dot{x}(t)=f(t, x), t \neq \tau_{k}, \\
& \Delta x=I_{k}(x), t=\tau_{k}, \\
& x\left(t_{0}^{+}\right)=x_{0},
\end{aligned}
$$

where $f: \mathbf{R}^{+} \times \Omega \rightarrow \mathbf{R}^{n}, I_{k}: \Omega \rightarrow \mathbf{R}^{n}, \Omega \subset \mathbf{R}^{n}$ is an open subset and $0=\tau_{-1}<\tau_{0}<\tau_{1}<\cdots$, with $\lim _{k \rightarrow \infty} \tau_{k}=+\infty$, as usual $\Delta x(t)=x\left(t^{+}\right)-x(t), x\left(t^{+}\right)$denote the right limit of $x$ at $t$. Then, we compare this method with $\theta$-method and Runge-kutta method, where discussed in [13].

Definition 1.1 (Baniov and Simeonov[2]) The function $x(t), t \in\left(t_{0}, b\right)$ is said to be the solution of the system with impulsive effect (1.1), if the following conditions are satisfied:

1. $x\left(t_{0}^{+}\right)=x_{0},(t, x(t)) \in \mathrm{R}^{+} \times \Omega$ for $t \in\left(t_{0}, b\right)$,

2. for $t \in\left(t_{0}, b\right), t \neq \tau_{k}, k \in \mathrm{N}$, the function $x(t)$ is differentiable and

$$
\left.\frac{d x(t}{d t}\right)=f(t, x(t)) \text {, }
$$

3. the function $x(t)$ is left continuous in $\left(t_{0}, b\right)$, if $t \in\left(t_{0}, b\right)$ and

$$
t=\tau_{k}, t \neq b \text {, then } x\left(t^{+}\right)=x(t)+I_{k}(x(t)) .
$$

Theorem $1.2([14,13])$ If $f: R^{+} \times \Omega \rightarrow R^{n}$ is continuous in $\left(\tau_{k}, \tau_{k+1}\right] \times \Omega, k \in N$, $\lim _{(t, y) \rightarrow\left(\tau_{k}^{+}, x\right)} f(t, y)$ is finite and exists and $f$ is locally Lipschitz continuous with respect to $x$ in $R^{+} \times \Omega$, then the solution $x(t)$ of problem (1.1) is unique.

In this paper, we consider the following impulsive differential equation (1.2), evaluate an approximation of $x(t)$ at each subinterval $(k, k+1]$ by Adomian's method. 
$\dot{x}(t)=\alpha x, t \neq k, t>0$,

$\Delta x=\beta x, t=k$,

$x\left(0^{+}\right)=x_{0}$,

where $\alpha \neq 0, \beta, x_{0} \in \mathrm{R}, 1+\beta \neq 0, k \in \mathrm{N}$.

Definition $1.3([6,13]) x(t)$ is said to be the solution of (1.2) if it satisfies the following conditions:

1. $\lim _{t \rightarrow 0^{+}} x(t)=x_{0}=x\left(0^{+}\right)$,

2. for $t \in(0,+\infty), t \neq k, k \in N, x(t)$ is differentiable and $\dot{x}(t)=\alpha x(t)$,

3. $x(t)$ is left continuous in $(0,+\infty)$ and if $t=k$, then $x\left(k^{+}\right)-x(k)=\beta x(k)$ , where $x\left(k^{+}\right)=\lim _{t \rightarrow k^{+}} x(t)$.

Problem (1.2), in $(0, \infty)$, has a unique solution

$x(t)=x_{0} e^{\alpha\{t\}}\left((1+\beta) e^{\alpha}\right)^{[t]}$,

where $[\mathrm{t}]$ and $\{t\}$ denote the greatest-integer function of $\mathrm{t}$ and the fractional part of $\mathrm{t}$, respectively.

Definition 1.4 ([13]) The solution $x(t)$ of Eq.(1.2) is asymptotically stable if $x(t) \rightarrow 0$ as $t \rightarrow \infty$.

From (1.3), it is easy to obtain the following theorem:

\section{Theorem 1.5}

The solution $x \equiv 0$ of Eq. (1.2) is asymptotically stable if and only if $\left|(1+\beta) e^{\alpha}\right|<1$.

\section{Method of Solution}

Consider the impulsive differential equation (1.2).

by integrating from $(k, k+1]$, we have:

$x(t)=x(0)+\alpha \int_{0}^{t} x(s) d s, \quad t \in(0,1]$,

and

$x(t)=x\left(k^{+}\right)+\alpha \int_{k}^{t} x(s) d s, t \in(k, k+1], k=1,2,3, \cdots$

by impulsive effect, we have:

$\Delta x(t)=x\left(k^{+}\right)-x(k)=\beta x(k)$, then $x\left(k^{+}\right)=(\beta+1) x(k)$, and thus 
$x(t)=(\beta+1) x(k)+\alpha \int_{k}^{t} x(s) d s, t \in(k, k+1], k=1,2,3, \cdots$

We define $\quad x_{k}(t)=x(t), t \in(k, k+1], k=0,1,2, \cdots \quad$ and $\quad x(0)=x_{0}$.

To solve (2.1) and (2.2) by Adomian's method, let

$x_{k}(t)=\sum_{n=0}^{\infty} x_{k, n}(t), k=0,1,2, \cdots$.

by substituting (2.3) in (2.1) and (2.2) gives

$x_{0}(t)=x_{0}+\alpha \int_{0}^{t} x_{0}(s) d s$

$x_{k}(t)=(\beta+1) x_{k-1}(k)+\alpha \int_{k}^{t} x_{k}(s) d s, t \in(k, k+1], k=1,2,3, \cdots$.

where $x_{k-1}(k)$ is an approximation of $x(k)$, therefore,

$\sum_{n=0}^{\infty} x_{0, n}(t)=x_{0}+\alpha \int_{0}^{t}\left(\sum_{n=0}^{\infty} x_{0, n}(s)\right) d s$

$\sum_{n=0}^{\infty} x_{k, n}(t)=(\beta+1) x_{k-1}(k)+\alpha \int_{k}^{t}\left(\sum_{n=0}^{\infty} x_{k, n}(s)\right) d s, \quad t \in(k, k+1], k=1,2,3, \cdots$.

these identity are satisfied if we set:

$x_{0,0}(t)=x_{0}$,

$x_{k, 0}(t)=(\beta+1) x_{k-1}(k), k=1,2,3, \cdots$.

$x_{k, n+1}(t)=\alpha \int_{k}^{t} x_{k, n}(s) d s, n=0,1,2, \cdots, k=0,1,2, \cdots$.

and,

$x(t)=x_{k}(t), t \in(k, k+1], k=0,1,2, \cdots$.

Relationship (2.3) gives an approximate analytical solution which converges perfectly towards the exact solution in the limit where $n \rightarrow \infty$ [15-16]. An error estimation can generally be given.

\section{$3 \quad$ Numerical Results}

In this section, We consider some first order impulsive differential equations. The following examples will be helpful to illustrate the main results of this paper.

Example 3.1 Consider the following impulsive differential equation:

$\left\{\begin{array}{l}\dot{x}(t)=-5 x, t \neq k, t>0, \\ \Delta x=-80 x, t=k, \\ x\left(0^{+}\right)=10,\end{array}\right.$

which has the analytic solution in the following form

$x(t)=x_{0} e^{-5\{t\}}\left((1+(-80)) e^{-5}\right)^{[t]}$, 
We know that, the exact solution of (3.1) is asymptotically stable,because $\left|(1+\beta) e^{\alpha}\right|=79 e^{-5}<1$.

if $\theta$-method with $\theta=1$ is applied to ODE, the numerical solution should be convergent. However, for impulsive differential equation (3.1), let $\theta=1$ and $h=1 / m(m=15)$, the numerical solution of (3.1) is divergent [13]. This example indicates that the $\theta$-method keeps the order of convergence but some properties have changed for system (1.2). Applying the numeric method (2.4), we have:

$$
x(t) \approx\left\{\begin{array}{l}
10-50 t+125 t^{2}-208.333 t^{3}+260.417 t^{4}-260.417 t^{5}+\cdots \quad, 0 \leq t \leq 1 \\
-790.89+3954.45 t-9886.11 t^{2}+16476.8 t^{3}-20595.7 t^{4}+\cdots, 1<t \leq 2 \\
62451.4-311673 t+776265 t^{2}-1.28453 \times 10^{6} t^{3}+\cdots, 2<t \leq 3 \\
-4.53661 \times 10^{6}+2.16489 \times 10^{7} t-5.06749 \times 10^{7} t^{2}+\cdots, 3<t \leq 4 \\
\vdots
\end{array}\right.
$$

and so on.

Figure 1, shows the numerical solution and exact solution of (3.1), in which the dashed curve is numerical solution using ADM, the solid curve is obtained by analytical expression (1.3).

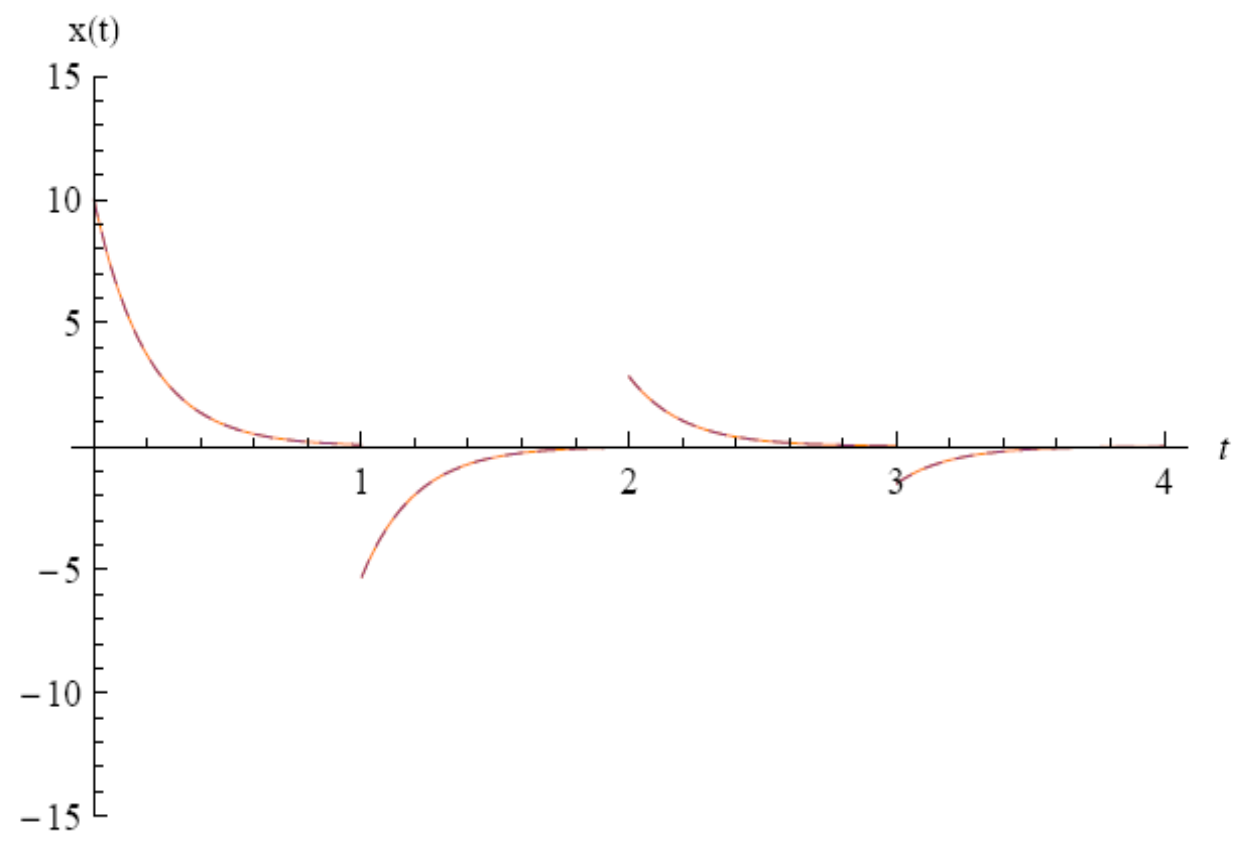

Fig. 1. The dashed curve is the numerical solution, the solid is the analytical solution of (3.1).

Example 3.2 Consider the following impulsive differential equation: 
$\left\{\begin{array}{l}\dot{x}(t)=1.2 x, t \neq k, t>0 \\ \Delta x=-0.9 x, t=k, \\ x\left(0^{+}\right)=1\end{array}\right.$

we obtain

$x_{1}(t)=1+1.2 t+0.72 t^{2}+0.288 t^{3}+0.0864 t^{4}+0.020736^{5}+\cdots$

and

$x(t)=\left\{\begin{array}{lc}x_{1}(t), & 0 \leq t \leq 1 \\ 0.1 x_{1}(t), & 1<t \leq 2 \\ 0.01 x_{1}(t), & 2<t \leq 3 \\ 0.001 x_{1}(t), & 3<t \leq 4 \\ \vdots & \end{array}\right.$

it shows that $x(t) \rightarrow 0$ as $t \rightarrow \infty$ and then the solution is stable.

Figure 2, shows the numerical solution and exact solution of (3.2), in which the dashed curve is numerical solution using ADM, the solid curve is obtained by analytical expression (1.3).

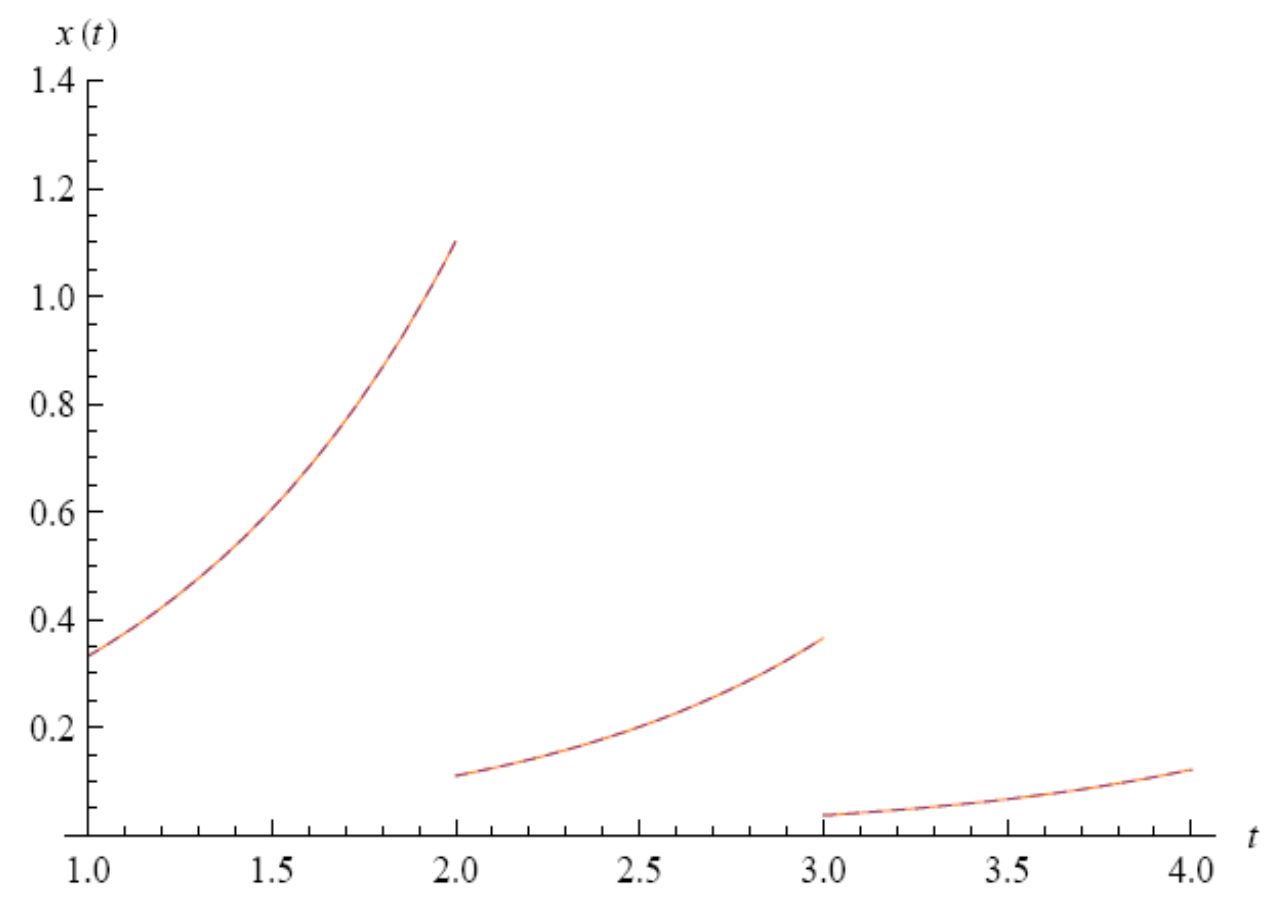

Fig. 2. The dashed curve is the numerical solution, the solid is the analytical solution of (3.2).

Example 3.3 Consider the following impulsive differential equation:

$$
\left\{\begin{array}{l}
\dot{x}(t)=-3.9 x, t \neq k, t>0 \\
\Delta x=10 x, t=k \\
x\left(0^{+}\right)=3
\end{array}\right.
$$


with applying the above method, we have:

$$
x(t)=\left\{\begin{array}{l}
3-11.7 t+22.815 t^{2}-29.6595 t^{3}+29.918 t^{4}-22.556 t^{5}+\cdots, 0 \leq t \leq 1 \\
33.0001-128.7 t+250.996 t^{2}-326.255 t^{3}+318.099 t^{4}-\cdots, 1<t \leq 2 \\
362.977-1415.45 t+2759.29 t^{2}-3584.46 t^{3}+3488.94 t^{4}-\cdots, 2<t \leq 3 \\
3957.21-15310.4 t+29446.5 t^{2}-37417.2 t^{3}+35187 t^{4}-\cdots, 3<t \leq 4 \\
\vdots
\end{array}\right.
$$

Figure 3, shows the numerical solution and exact solution of (3.3), in which the dashed curve is numerical solution using ADM, the solid curve is obtained by analytical expression (1.3).

\section{Conclusion}

Example (3.1) indicates that the $\theta$ - method with $\theta=1$ and $h=1 / m(m=15)$ is divergent [13]. The coefficient of problem (3.2) is $\alpha=1.2$, which is greater than zero. According to [13], we must choose $\theta$ - methods, 3-Lobatto IIIB and 2-Radau IA methods to obtain the numerical solution of (3.2) with stepsize $h=1 / \mathrm{m}$.

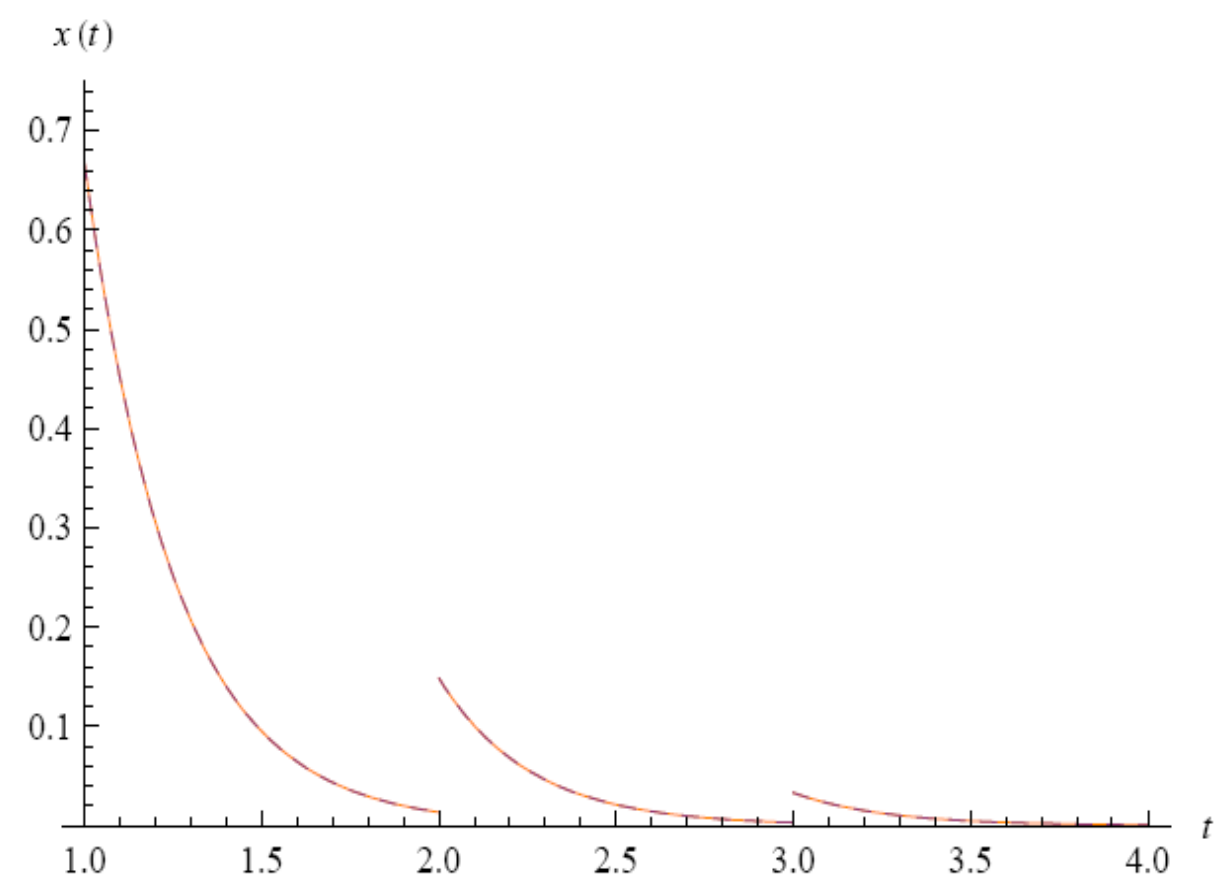

Fig. 3. the dashed curve is the numerical solution, the solid is the analytical of (3.3), solution.

In example (3.3), we observed that $\alpha=-3.9<0$, so the 2-Lobatto IIIB and 3-Gauss methods must applied.

But, In this work, we introduced the ADM for all of this problems, whether $\alpha<0$ or $\alpha>0$ and give very best approximation. In Table 1 , we calculate the relative errors (RE) 
and absolute errors (AE) for Ex.(3.1) at $t=3,4,5$. It shows that this method is convergent. The exact solutions of (3.2) at $t=8$ and (3.3) at $t=4$ are $x \approx 1.215104 e-001$, $x \approx 6.70356 e-004$, respectively. In order to obtain convergent solution for (3.2), $\theta$ must satisfy $0 \leq \theta \leq 0.4180233$, and for (3.3) $0 \leq \theta \leq 0.53$ [13]. In Tables $2-5$ the relative errors (RE) and absolute errors (AE) are listed. In Tables 6 and 7, we calculated the relative errors (RE) and absolute errors (AE) for (3.2) and (3.3) by Adomian Decomposition Method.

Table 1: Errors of problem (3.1) by ADM. at $t=3,4,5$.

\begin{tabular}{||l|rl|}
\hline $\mathrm{t}$ & $A E$ & $R E$ \\
\hline 3 & $6.46158 \mathrm{e}-005$ & $3.38456 \mathrm{e}-003$ \\
4 & $4.58857 \mathrm{e}-005$ & $4.51529 \mathrm{e}-003$ \\
5 & $3.05483 \mathrm{e}-005$ & $5.64729 \mathrm{e}-003$ \\
\hline \hline
\end{tabular}

Table 2: Errors of problem (3.2) at $t=8$ by $\theta-$ method

\begin{tabular}{||c|cc|cc|}
\hline & \multicolumn{2}{|c|}{$\theta=0$} & \multicolumn{2}{c|}{$\theta=0.4$} \\
\hline $\mathrm{m}$ & $A E$ & $R E$ & $A E$ & $R E$ \\
\hline 2 & $7.856074 \mathrm{e}-002$ & $6.465351 \mathrm{e}-001$ & $1.636205 \mathrm{e}-002$ & $1.346555 \mathrm{e}-001$ \\
20 & $1.571442 \mathrm{e}-002$ & $1.293257 \mathrm{e}-001$ & $3.262087 \mathrm{e}-003$ & $2.684615 \mathrm{e}-002$ \\
100 & $3.422626 \mathrm{e}-003$ & $2.816734 \mathrm{e}-002$ & $6.901199 \mathrm{e}-004$ & $5.679512 \mathrm{e}-003$ \\
200 & $1.730344 \mathrm{e}-003$ & $1.424029 \mathrm{e}-002$ & $3.474957 \mathrm{e}-004$ & $2.859802 \mathrm{e}-003$ \\
\hline
\end{tabular}

Table 3: Errors of problem (3.3) at $t=4$ by $\theta-$ method

\begin{tabular}{||c|rl|cl|}
\hline & \multicolumn{2}{|c|}{$\theta=0.2$} & \multicolumn{2}{c|}{$\theta=0.5$} \\
\hline $\mathrm{m}$ & $A E$ & $R E$ & $A E$ & $R E$ \\
\hline 8 & $6.428826 \mathrm{e}-004$ & $9.590170 \mathrm{e}-001$ & $1.838000 \mathrm{e}-004$ & $2.741827 \mathrm{e}-001$ \\
16 & $4.928432 \mathrm{e}-004$ & $7.351964 \mathrm{e}-001$ & $5.025963 \mathrm{e}-005$ & $7.497455 \mathrm{e}-002$ \\
100 & $1.141830 \mathrm{e}-004$ & $1.703320 \mathrm{e}-001$ & $1.324487 \mathrm{e}-006$ & $1.975797 \mathrm{e}-003$ \\
500 & $3.989841 \mathrm{e}-009$ & $3.600510 \mathrm{e}-002$ & $5.301817 \mathrm{e}-008$ & $7.908959 \mathrm{e}-005$ \\
\hline
\end{tabular}

Table 4: Errors of problem (3.2) at $t=8$ by higher-order Runge-Kutta methods

\begin{tabular}{||c|cl|cl|}
\hline & \multicolumn{2}{|c|}{ 3-Lobatto IIIB } & \multicolumn{2}{c|}{ 2-Radau IA } \\
\hline $\mathrm{m}$ & $A E$ & $R E$ & $A E$ & $R E$ \\
\hline 2 & $1.071861 \mathrm{e}-004$ & $8.821141 \mathrm{e}-004$ & $6.253585 \mathrm{e}-003$ & $1.715514 \mathrm{e}-002$ \\
5 & $2.696801 \mathrm{e}-006$ & $2.219399 \mathrm{e}-005$ & $3.594719 \mathrm{e}-004$ & $9.861209 \mathrm{e}-004$ \\
10 & $1.681199 \mathrm{e}-007$ & $1.383584 \mathrm{e}-006$ & $4.340316 \mathrm{e}-005$ & $1.190657 \mathrm{e}-004$ \\
20 & $1.050075 \mathrm{e}-008$ & $8.641851 \mathrm{e}-008$ & $5.335311 \mathrm{e}-006$ & $1.463609 \mathrm{e}-005$ \\
40 & $6.561920 \mathrm{e}-010$ & $5.400295 \mathrm{e}-009$ & $2.204902 \mathrm{e}-007$ & $1.814579 \mathrm{e}-006$ \\
\hline
\end{tabular}

Table 5: Errors of problem (3.3) at $t=4$ by higher-order Runge-Kutta methods

\begin{tabular}{||c|cl|cl|}
\hline & \multicolumn{2}{|c|}{ 2-Lobatto IIIB } & \multicolumn{2}{c|}{3 -Gauss } \\
\hline $\mathrm{m}$ & $A E$ & $R E$ & $A E$ & $R E$ \\
\hline 5 & $3.900707 \mathrm{e}-004$ & $5.818861 \mathrm{e}-001$ & $2.392048 \mathrm{e}-008$ & $3.568325 \mathrm{e}-005$ \\
10 & $1.228127 \mathrm{e}-004$ & $1.832053 \mathrm{e}-001$ & $3.672177 \mathrm{e}-010$ & $5.477952 \mathrm{e}-007$ \\
20 & $3.251276 \mathrm{e}-005$ & $4.850075 \mathrm{e}-002$ & $5.712409 \mathrm{e}-012$ & $8.521458 \mathrm{e}-009$ \\
40 & $8.245050 \mathrm{e}-006$ & $1.229951 \mathrm{e}-002$ & $8.916153 \mathrm{e}-014$ & $1.330063 \mathrm{e}-010$ \\
100 & $1.324487 \mathrm{e}-006$ & $1.975797 \mathrm{e}-003$ & $3.713392 \mathrm{e}-016$ & $5.539435 \mathrm{e}-013$ \\
\hline
\end{tabular}


Table 6: Errors of problem (3.2) by ADM. at $t=6,7,8$.

\begin{tabular}{||l|rl|}
\hline $\mathrm{t}$ & $A E$ & $R E$ \\
\hline 6 & $2.81858 \mathrm{e}-014$ & $2.10431 \mathrm{e}-012$ \\
7 & $2.91708 \mathrm{e}-013$ & $6.55955 \mathrm{e}-011$ \\
8 & $8.62303 \mathrm{e}-013$ & $5.84027 \mathrm{e}-010$ \\
\hline
\end{tabular}

Table 7: Errors of problem (3.3) by ADM. at $t=3,3.5,4$

\begin{tabular}{||c|cl|}
\hline $\mathrm{t}$ & $A E$ & $R E$ \\
\hline 3 & $1.85422-008$ & $6.15886 \mathrm{e}-006$ \\
3.5 & $3.19589 \mathrm{e}-008$ & $6.78285 \mathrm{e}-006$ \\
4 & $1.22828 \mathrm{e}-008$ & $1.83229 \mathrm{e}-005$ \\
\hline
\end{tabular}

\section{References}

[1] D.D. Bainov, A. Dishliev, Population dynamics control in regard to minimizing the time necessary for the regeneration of a biomass taken away from the population, Comptes rendus de l'Academie Bulgare des Sciences, 42 (12) (1989) 29--32.

[2] D.D. Bainov, P.S. Simenov, Systems with Impulse Effect Stability Theory and Applications, Ellis Horwood Limited, Chichester, 1989.

[3] A. Dishliev, D.D. Bainov, Dependence upon initial conditions and parameters of solutions of impulsive differential equations with variable structure, International Journal of Theoretical Physics, 29 (6) (1990) 655--676.

[4] V.D. Mil'man, A.D. Myshkis, On the stability of motion in the presence of impulses, Sib. Math. J., 1 (1960) 233--237.

[5] M.U. Akhmet, On the general problem of stability for impulsive differential equations, J. Math. Anal. Appl., 288 (2003) 182--196.

[6] D.D. Bainov, P.S. Simeonov, Systems With Impulsive Effect: Stability, Theory and Applications, Ellis Horwood, Chichester, 1989.

[7] L.Z. Dong, L. Chen, L.H. Sun, Extiction and permanence of the predator-prey system with stocking of prey and harvesting of predator impulsively, Math. Meth. Appl. Sci., 29 (2006) 415--425.

[8] V. Lakshmikantham, D.D. Bainov, P.S. Simeonov, Theory of Impulsive Differential Equations, World Scientific, Singapore, 1989.

[9] A.M. Samoilenko, N.A. Perestyuk, Impulsive Differential Equations, World Scientific, Singapore, 1995. 
[10] G. Kulev, D.D. Bainov, On the stability of systems with impulsive by sirect method of Lyapunov, J. Math. Anal. Appl., 140 (1989) 324--340.

[11] D.D. Bainov, G. Kulev, Application of Lyapunov's functions to the investigation of global stability of solutions of system with impulses,Appl. Anal., 26 (1) (1988) 255--270.

[12] B.M. Randelovic, L.V. Stefanovic, B.M. Dankovic, Numerical solution of impulsive differential equations, Facta Univ. Ser. Math. Inform., 15 (2000) 101--111.

[13] X. J. Ran, M. Z. Liu, Q. Y. Zhu, Numerical methods for impulsive differential equation, Math. and Computer Modelling.,48 (2008) 46--55.

[14] X.L. Fu, B.Q. Yan, Introduction to the Impulsive Differential System, Scientific Publishers, 2005, pp. 1--33 (in Chinese).

[15] Y. Cherruault, Mode'les et Me'thodes Mathe'matiques pour les Sciences du Vivant, Presses Universitaires de France, 1998.

[16] Y. Cherruault, G. Adomian, Decomposition methods: a new proof of convergence, Math. Comput. Model., 18 (1993)103--106.

[17] K. Abbaoui, Y. Cherruault, Convergence of Adomian's method applied to differential equations, Comput. Math. Appl., 28(1994) 103--109. 